Name: Peer Review Information for "<i>Operando </i>Electrochemical X-ray Diffraction and Raman Spectroscopic Studies Reveal the Alkali-Metal Ion Intercalation Mechanism in Prussian Blue Analogues"

First Round of Reviewer Comments

Reviewer: 1

Comments to the Author

This manuscript reports on operando X-ray and Raman spectroscopic observation of the alkali-metal ion intercalation process in Berlin green, which demonstrated the different intercalation mechanisms for K+ and $\mathrm{Na}+$. This work is a good example of in situ combined spectroscopy and publishable in the journal after minor revisions.

1. In Figs. 3a, 3d, 4a, an $4 d$, the range of the $y$-axes seems to be incorrect; although the values of $x$ monotonically increase during the cyclic potential change, this is apparently impossible. The range should be denoted as " $0-1.27-0$ " like the $x$-axis of Fig. 5 a. According to Eq. (2), the value of $x$ should be less than 2 .

2. What is the meaning of $v<\sup >2</$ sup $>$ ?

3. In page 4 , the authors stated that the $v<\sup >2</$ sup $>(\mathrm{Fe}-\mathrm{C})$ is more sensitive than the $v<\sup >2</$ sup $>(C \equiv N)$ to intercalation amount. However, Fig. 5a seems to be opposite.

Reviewer: 2

Comments to the Author

In this manuscript, Li et al reports the study of the $\mathrm{Na}+$ and $\mathrm{K}+$ intercalation mechanism in Prussian Blue analogues (PBA). The authors have developed an electrochemical cell that can conduct in situ XRD and Raman measurement. Through delicate characterizations and data interpretations, the different intercalation behavior of $\mathrm{Na}+$ and $\mathrm{K}+$ has been revealed and a new strategy using a $\mathrm{Na}+/ \mathrm{K}+$ hybrid cation 
electrolyte to realize both high voltage and energy density of the PBA has been proposed. These results are very interesting and I believe that the mechanism studies can provide valuable insight to guide the design of PBA for aqueous rechargeable metal-ion batteries. Therefore, I recommend the acceptance of this manuscript for publication in The Journal of Physical Chemistry Letters after minor changes as noted.

1. While the in situ XRD and Raman results reveal the different intercalation mechanism (solid solution vs. two-phase reaction) between $\mathrm{Na}+$ and $\mathrm{K}+$, what is the fundamental origin for such differences? I suggest the authors to discuss this issue in the revision.

2. It is very interesting that using a $\mathrm{Na}+/ \mathrm{K}+$ hybrid cation electrolyte can realize both high voltage and energy density of the PBA. It is $\mathrm{Na}+/ \mathrm{K}+$ co-intercalation or $\mathrm{Na}+$ or $\mathrm{K}+$ dominate? Any experimental results to support?

3. Phase delay is often seen in in situ experiment due to the design flaw of the in situ cell (S.Bak et al. NPG Asia Materials. 2018, 10, 563). I would suggest the analysis of the in situ XRD (e.g. lattice parameters) and compare with the ex situ result (for example, fully charged or discharged states).

Reviewer: 3

\section{Comments to the Author}

In this work, the authors aimed to distinguish the insertion of $\mathrm{Na}+$ and $\mathrm{K}+$ into Berlin Green in aqueous metal-ion batteries. The authors applied multiple in situ techniques, namely XRD and Raman spectroscopy, to investigate the influence of the mobile ion on the structural evolution and local symmetry of Fe, respectively. In spite of the interesting approach, several major points need to be addressed before this paper can be considered for publication

1. The use of Berlin Green in aqueous potassium system is already known in the literature (see for instance ref. [1])

2. The insertion mechanism observed in the BG is somehow weird. Previous works on the same material in aqueous environments [2] clearly show the occurrence of two plateaus at 0.8 and $0.2 \mathrm{~V}$ vs. SHE, corresponding to the oxidation/reduction of low-spin iron (bonded to the C-side of cyanide) and high-spin iron (bonded to the $\mathrm{N}$ side of the cyanide). In the compound shown here, the high voltage plateau (which is not discussed in the text) is extremely short. Why? Which is the crystallinity/purity of the material? The amount of defects? Without this information, and a thorough characterization of the pristine material, the rest of the study is meaningless.

3. Raman spectroscopy is used to characterize the oxidation state of the iron. However, since there are two different iron sites which may undergo oxidation/reaction; Raman spectroscopy cannot therefore be so simply applied. The authors should base their interpretation of the Raman spectra on the more complete work of Samain et al. [3], who applied Raman spectroscopy together with Mössbauer spectroscopy in order to really understand the state of the iron in similar compounds. 
4. The authors state that the $\mathrm{Na}+$ insertion caused a solid-solution mechanism with the structure transforming from cubic to rhombohedral. This is somewhat strange, since a solid-solution is usually occurring without a phase transition. Later on, a few lines later (page 3, left column, line 54-55), the authors contradict themselves describing the observation as a phase transition. Moreover, according to Figure 3, there seem to be no phase transition in the case of $\mathrm{Nat}$, while there might be a bi-phasic behavior in the case of $\mathrm{K}+$.

5. We don't agree with the conclusion about the unchanged $v(C \equiv N)$ when using K2SO4. If you look at the region between 2100 and $2200 \mathrm{~cm}-1$, there is a band increasing in inteisty at ca. $2130 \mathrm{~cm}-1 \mathrm{from}$ the middle of the discharge. The strong peak at ca. $2170 \mathrm{~cm}-1$ shifts to lower frequencies when approaching the end of discharge. A much deeper and detailed interpretation on the modification of the $v(C \equiv N)$ vibration is expected.

6. The conclusion about that the $\mathrm{v} 2(\mathrm{Fe}-\mathrm{C})$ is more sensitive than $\mathrm{v} 2(\mathrm{C} \equiv \mathrm{N})$ conflicted with Figure $5 \mathrm{a}$ regarding to the case of $\mathrm{Na}+$.

7. We think that the authors should compare the Raman spectra of the pristine BG with those at the EOD (with $\mathrm{Na}+$ and $\mathrm{K}+$ ) to better explain how the change in the symmetry influence on the mechanism.

8. The conclusion about volume expansion due to the $\mathrm{K}+$ insertion on page 6 , right column, line 51 52 conflicted with the operando XRD (Figure 3e,f), where we see the peaks shifting to the higher angles, which rather indicate a reduction of the lattice size.

\section{Minor comments:}

1. According to the common scientific terminology, "intercalation" is the insertion of molecules or ions into a layered material. Since BG is not layered material, "insertion" should be used instead. This should be corrected everywhere in the paper.

2. In the Figures S5, S6, S8, separate the diffractograms as in Figure S7.

\section{References}

[1] M. Wang, H. Wang, H. Zhang, X. Li, Aqueous K-ion battery incorporating environment-friendly organic compound and Berlin green, J. Energy Chem. 48 (2020) 14-20.

doi:10.1016/j.jechem.2019.12.019.

[2] W. Shi, P. Nie, X. Shang, J. Yang, Z. Xie, R. Xu, J. Liu, Berlin green-based battery deionizationhighly selective potassium recovery in seawater, Electrochim. Acta. 310 (2019) 104-112.

doi:10.1016/j.electacta.2019.04.122. 
[3] L. Samain, B. Gilbert, F. Grandjean, G.J. Long, D. Strivay, Redox reactions in Prussian blue containing paint layers as a result of light exposure, J. Anal. At. Spectrom. 28 (2013) 524. doi:10.1039/c3ja30359d.

\section{Author's Response to Peer Review Comments:}

$\underline{\text { Review Comments (in black) and Responses (in blue) }}$

\section{Reviewer 1:}

Recommendation: This paper is publishable subject to minor revisions noted. Further review is not needed.

Comments:

This manuscript reports on operando X-ray and Raman spectroscopic observation of the alkali-metal ion intercalation process in Berlin green, which demonstrated the different intercalation mechanisms for $\mathrm{K}^{+}$and $\mathrm{Na}^{+}$. This work is a good example of in situ combined spectroscopy and publishable in the journal after minor revisions.

1. In Figs. 3a, 3d, $4 a$, an $4 d$, the range of the y-axes seems to be incorrect; although the values of $x$ monotonically increase during the cyclic potential change, this is apparently impossible. The range should be denoted as " 0 - 1.27 - 0" like the x-axis of Fig. 5a. According to Eq. (2), the value of $\mathrm{x}$ should be less than 2.

Response: We thank the reviewer for pointing out this problem. In fact, the way in our manuscript (Fig. $3 \mathrm{a}, 3 \mathrm{~b}, 4 \mathrm{a}$ and $4 \mathrm{~d}$ ) to present the intercalation amount of ions vs. potential, where the intercalation value monotonically increases in the y-axes, is widely used in the literature. We agree with reviewer that the intercalation amount should be less than 2 in principle. We have modified range of the y-axes as " $0-1.27$ - 0 " for $\mathrm{Na}^{+}$and " 0 - $0.97-0$ " for $\mathrm{K}^{+}$in Fig. 3a, 3d, 4a, and 4d.

2. What is the meaning of $v<\sup >2</$ sup $>$ ?

Response: We are sorry about the confusion. But we can not find any expression of " $v<\sup >2</$ sup $>$ " throughout the version of manuscript and supporting information on the submission system. Here the " $v<\sup >2</$ sup $>$ " should mean " $v$ ".

3. In page 4 , the authors stated that the $v<\sup >2</$ sup $>(\mathrm{Fe}-\mathrm{C})$ is more sensitive than the $v<\sup >2</$ sup $>(C \equiv N)$ to intercalation amount. However, Fig. 5a seems to be opposite.

Response: Thanks for pointing out this issue. As shown in Fig. 5a, the dependence of $v^{2}(\mathrm{Fe}-\mathrm{C})$ on the intercalation amount of cations in $\mathrm{Na}_{2} \mathrm{SO}_{4}$ is similar to that in $\mathrm{K}_{2} \mathrm{SO}_{4}$, while the dependence of $v^{2}(\mathrm{C} \equiv \mathrm{N})$ is obviously different. We propose that $\mathrm{Fe}$ in $\mathrm{BG}$ gains electrons first during the intercalation process, resulting in the shift of the $v(\mathrm{Fe}-\mathrm{C})$ peak. Since the gain or loss of electrons only relate to the change of bond order of Fe-C, the curves of $v(\mathrm{Fe}-\mathrm{C})$ in $\mathrm{Na}_{2} \mathrm{SO}_{4}$ and $\mathrm{K}_{2} \mathrm{SO}_{4}$ overlap with each other well. After the ion intercalation, the lattice structure of BG changes. The different dependence of $v(C \equiv N)$ suggests different intercalation mechanisms for $\mathrm{Na}^{+}$and $\mathrm{K}^{+}$. As $\mathrm{Fe}$ is the redox center, it is more precise to say that $v^{2}(\mathrm{Fe}-\mathrm{C})$ is more sensitive to the gain or loss of electron than the $v^{2}(\mathrm{C} \equiv \mathrm{N})$. We have modified the description in line 23-26 and line 48 to 53 (left column) in page 4 as follows:

We then plot the amount of intercalated cations with $v^{2}(\mathrm{Fe}-\mathrm{C})$ at $539 \mathrm{~cm}^{-1}$ to those of $v^{2}(\mathrm{C} \equiv \mathrm{N})$ at $2158 \mathrm{~cm}^{-1}$. As shown in Fig. 5a, the dependence of $v^{2}(\mathrm{Fe}-\mathrm{C})$ on the intercalation amount of cations in $\mathrm{Na}_{2} \mathrm{SO}_{4}$ is similar 
to that in $\mathrm{K}_{2} \mathrm{SO}_{4}$, while the dependence of $v^{2}(\mathrm{C} \equiv \mathrm{N})$ is obviously different. We propose that $\mathrm{Fe}$ in $\mathrm{BG}$ gains electrons first during the intercalation process, resulting in the shift of the $v(\mathrm{Fe}-\mathrm{C})$ peak. Since the gain or loss of electrons only relate to the change of bond order of $\mathrm{Fe}-\mathrm{C}$, the curves of $v(\mathrm{Fe}-\mathrm{C})$ in $\mathrm{Na}_{2} \mathrm{SO}_{4}$ and $\mathrm{K}_{2} \mathrm{SO}_{4}$ overlap with each other well. After the ion intercalation, the lattice structure of $\mathrm{BG}$ changes. The different dependence of $v(C \equiv N)$ suggests different intercalation mechanisms for $\mathrm{Na}^{+}$and $\mathrm{K}^{+}$.

\section{Reviewer 2:}

Recommendation: This paper is publishable subject to minor revisions noted. Further review is not needed.

Comments:

In this manuscript, $\mathrm{Li}$ et al reports the study of the $\mathrm{Na}+$ and $\mathrm{K}+$ intercalation mechanism in Prussian Blue analogues (PBA). The authors have developed an electrochemical cell that can conduct in situ XRD and Raman measurement. Through delicate characterizations and data interpretations, the different intercalation behavior of $\mathrm{Na}+$ and $\mathrm{K}+$ has been revealed and a new strategy using a $\mathrm{Na}+/ \mathrm{K}+$ hybrid cation electrolyte to realize both high voltage and energy density of the PBA has been proposed. These results are very interesting and I believe that the mechanism studies can provide valuable insight to guide the design of PBA for aqueous rechargeable metal-ion batteries. Therefore, I recommend the acceptance of this manuscript for publication in The Journal of Physical Chemistry Letters after minor changes as noted.

1. While the in situ XRD and Raman results reveal the different intercalation mechanism (solid solution vs. two-phase reaction) between $\mathrm{Na}^{+}$and $\mathrm{K}^{+}$, what is the fundamental origin for such differences? I suggest the authors to discuss this issue in the revision.

Reponse: The different intercalation mechanism between $\mathrm{Na}^{+}$and $\mathrm{K}^{+}$probably originate from the different radius of ions. As $\mathrm{Na}^{+}$is small, the framework of BG can still maintain after the intercalation of $\mathrm{Na}^{+}$, leading to a solid solution mechanism mainly. However, when the cation becomes larger in the case of $\mathrm{K}^{+}$, BG cannot keep its structure after insertion, leading to a phase change. We have added this discussion in the revised manuscript in line 21-27 (right column) in page 4 as follows:

With these results, we can now understand the different $\mathrm{CV}$ behaviors in $\mathrm{Na}^{+}$and $\mathrm{K}^{+}$solutions. The radius $\mathrm{Na}^{+}$is small, the framework of $\mathrm{BG}$ can still maintain after the intercalation of $\mathrm{Na}^{+}$. As a result, $\mathrm{Na}^{+}$ intercalation in BG follows a solid solution mechanism mainly, resulting in an s-shaped potential profile. The charging voltage gradually shifts positively with $\mathrm{Na}^{+}$deintercalation as a result of the concentration polarization, leading to a lower voltage. Whereas when the cation becomes larger in the case of $\mathrm{K}^{+}, \mathrm{BG}$ cannot keep its structure after $\mathrm{K}^{+}$inserted, leading to a phase change. Therefore, $\mathrm{K}^{+}$intercalation follows a two-phase transition mechanism with a stable voltage, in which the Gibbs free energy difference......

2. It is very interesting that using a $\mathrm{Na}+/ \mathrm{K}+$ hybrid cation electrolyte can realize both high voltage and energy density of the PBA. It is $\mathrm{Na}+/ \mathrm{K}+$ co-intercalation or $\mathrm{Na}+$ or $\mathrm{K}+$ dominate? Any experimental results to support?

Response: The substitution of various compositions and/or proportions of the two parts of transition metal and anion groups in a same structure type is usually use to improve the performance of cathode materials. In $\mathrm{BG}$, the inserted $\mathrm{K}^{+}$serve as the "pillar" in pristine structure to provide the corresponding larger cell volume for $\mathrm{Na}^{+}$insertion. From the XRD Rietveld refinement analysis of ex situ measurements for PBAs in non-aqueous solution, the $\mathrm{Na}+$ insertion dominates, which ensures a high specific capacity (Adv. Funct. Mater. 30 (2020) 1910840). In our in situ measurements, it is still a challenge to perform reliable Rietveld refinement analysis to extract the concentration of $\mathrm{Na}^{+}$and $\mathrm{K}^{+}$due to the relatively weak signal. 
3. Phase delay is often seen in in situ experiment due to the design flaw of the in situ cell (S.Bak et al. NPG Asia Materials. 2018, 10, 563). I would suggest the analysis of the in situ XRD (e.g. lattice parameters) and compare with the ex situ result (for example, fully charged or discharged states).

Response: The design of in situ cell is the first important step to perform in situ experiments successfully. Because BG is easily oxidized in air, we carefully seal the cell to avoid oxygen. We conducted ex situ measurements of $\mathrm{BG}$ after a discharge and charge cycle in $\mathrm{K}_{2} \mathrm{SO}_{4}$. Fig. R1 shows galvanostatic potential profile of BG (a), ex situ XRD pattern (b) and the zoom-in XRD pattern (c). The diffraction peaks of the initial state (1), reduced state (2) and fully charged state (3) are 17.495, 17.485 and 17.49 respectively, which shows an opposite change direction comparing with our in situ results.

Figure R1. (a) Galvanostatic potential profiles of $\mathrm{BG}$ electrode in $0.5 \mathrm{M} \mathrm{K}_{2} \mathrm{SO}_{4}$ at $1 \mathrm{C}$. (b) the corresponding in-situ XRD patterns and (c) the zoom-in XRD patterns

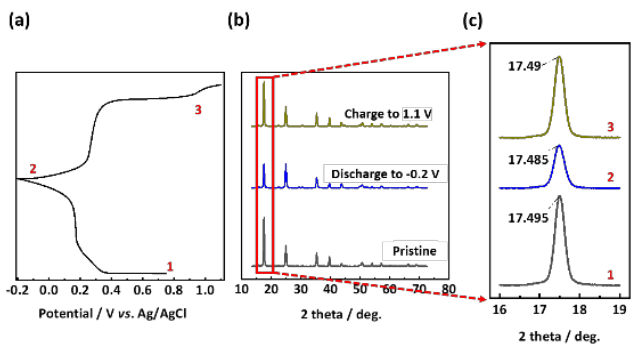

We have added the description of ex situ result in line xx:

Contrary to that in $\mathrm{Na}_{2} \mathrm{SO}_{4}$, all diffraction peaks move toward higher angles in $\mathrm{K}_{2} \mathrm{SO}_{4}$ (Figure $3 \mathrm{~d}$, e, and f), which was also confirmed by the potential-controlled electrochemical XRD study (Figure S7). Due to chemical changes induced by air and moisure, the ex situ results (Figure S8) shows an opposite change comparing with the in situ ones, demonstrating the importance of conducting in situ measurements. There are three views in the literature to explain this contradictory phenomenon: ......

\section{Reviewer 3:}

Recommendation: This paper is not recommended because it does not provide new physical insights

In this work, the authors aimed to distinguish the insertion of $\mathrm{Na}+$ and $\mathrm{K}+$ into Berlin Green in aqueous metal-ion batteries. The authors applied multiple in situ techniques, namely XRD and Raman spectroscopy, to investigate the influence of the mobile ion on the structural evolution and local symmetry of Fe, respectively. In spite of the interesting approach, several major points need to be addressed before this paper can be considered for publication

1. The use of Berlin Green in aqueous potassium system is already known in the literature (see for instance ref. [1])

Response: We agree with the reviewer that aqueous rechargeable metal-ion batteries have attracted great attention benefitted from their unique features for large scale energy storage technologies. Great efforts have been devoted to developing materials. For example, as the works (ref. 1) mentioned by the reviewer, the authors reported a new aqueous K-ion battery using environment-friendly 1,4,5,8naphthalenetetracarboxylic dianhydride-derived polyimide as anode and Berlin green as cathode. Both electrodes have high rate performance and excellent capacity retention during cycling. Attributing to wellmatched performance of cathode and anode materials, the full battery shows a high specific capacity, rate capability, and capacity retention. 
However, there are still few reports on optimizing cell performance from mechanistic understanding and studies. In our work, we focused on mechanistic study by developing home-built spectroelectrochemical cell for operando XRD and Raman spectroscopies, which allows us to obtain the correlated local structure, crystal structure, redox activity, and potential profiles during the charging and discharging processes. The combination of XRD and Raman techniques reveals that the intercalation of $\mathrm{Na}^{+}$and $\mathrm{K}^{+}$follows different mechanisms, which provides a new physical insight to clarify the long-term controversial understanding about the effect of the alkali insertion ion for PBAs. Furthermore, with this understanding, we propose a new strategy using a $\mathrm{Na}^{+} / \mathrm{K}^{+}$hybrid cation electrolyte to realize both high voltage and energy density. Our work also offers a unique insight for improving the cell performance from the understanding of the reaction mechanism.

2. The insertion mechanism observed in the BG is somehow weird. Previous works on the same material in aqueous environments [2] clearly show the occurrence of two plateaus at 0.8 and $0.2 \mathrm{~V}$ vs. SHE, corresponding to the oxidation/reduction of low-spin iron (bonded to the C-side of cyanide) and high-spin iron (bonded to the $\mathrm{N}$ side of the cyanide). In the compound shown here, the high voltage plateau (which is not discussed in the text) is extremely short. Why? Which is the crystallinity/purity of the material? The amount of defects? Without this information, and a thorough characterization of the pristine material, the rest of the study is meaningless.

Response: The different electrochemical behavior of BG in our work and the work the reviewer mentioned (Ref. 2: Electrochim. Acta., 310 (2019) 104) is mainly due to the crystallinity of the materials. BG nanoparticles were synthesized by a co-precipitation method in Ref. 2. Because the reaction between $\mathrm{Fe}^{3+}$ and $\mathrm{Fe}(\mathrm{CN})_{6}{ }^{3-}$ is very fast, the crystallinity of the as-prepared material is relatively low. As pointed out in the literature (Adv. Energy Mater., 5 (2015) 1401869), there are more vacancies in the BG with low crystallinity to accommodate metal ions, leading to a long high-voltage plateau. However, in our work BG were synthesized by a hydrolytic precipitation method using $\mathrm{Fe}(\mathrm{CN})_{6}^{3-}$ as a single iron-source (ACS Appl. Mater. Interfaces, 8 (2016) 23706), where the $\mathrm{Fe}(\mathrm{CN})_{6}{ }^{3-}$ anion decompose to $\mathrm{Fe}^{3+}$ in $\mathrm{HCl}$ first and then the $\mathrm{Fe}^{3+}$ ions coordinate with undecomposed $\mathrm{Fe}(\mathrm{CN})_{6}{ }^{3-}$ anions to form Prussian yellow $\mathrm{FeFe}(\mathrm{CN})_{6}$. Then $\mathrm{FeFe}(\mathrm{CN})_{6}$ is reduced and generate some $\mathrm{Fe}(\mathrm{CN})_{6}{ }^{4-}$ ions, which initiates the nucleation formation and the subsequent crystal growth. The as-prepared BG using single iron-source method possesses low defect, high crystallinity as demonstrated by the intense and sharp XRD peaks in Fig. 1a and perfect nanocubic morphology (Fig. 1a inset). In addition, the experimental conditions including the electrolyte, concentration and the scan rate are different between our works and Ref. 2. Therefore, it can be understood that the electrochemical behavior is different.

3. Raman spectroscopy is used to characterize the oxidation state of the iron. However, since there are two different iron sites which may undergo oxidation/reaction; Raman spectroscopy cannot therefore be so simply applied. The authors should base their interpretation of the Raman spectra on the more complete work of Samain et al. [3], who applied Raman spectroscopy together with Mössbauer spectroscopy in order to really understand the state of the iron in similar compounds.

Response: We agree with the reviewer that Mössbauer spectroscopy is a powerful tool to distinguish lowspin and high spin iron. As the work mentioned by the review (Ref. 3: J. Anal. At. Spectrom., 28 (2013) 524), the authors used Raman and Mössbauer spectroscopy to characterize the fading process of the paint layer consisted with Prussian blue and a white pigment under light exposure. As PBAs is unstable in air, an in situ electrochemical Mössbauer spectroscopy is required, which is a great challenge for us at present. In addition, in our work the variation of spectral features of Raman spectroscopy, in particular the variation of $\mathrm{C} \equiv \mathrm{N}$ peak, was used to illustrate the local structural change during charge and discharge processes. Combined with the in situ XRD characterization, we can conclude that the intercalation of $\mathrm{Na}^{+}$and $\mathrm{K}^{+}$ 
follows different mechanisms. We think the detailed oxidation state of the iron does not influence the scientific issue of the manuscript.

4. The authors state that the $\mathrm{Na}+$ insertion caused a solid-solution mechanism with the structure transforming from cubic to rhombohedral. This is somewhat strange, since a solid-solution is usually occurring without a phase transition. Later on, a few lines later (page 3, left column, line 54-55), the authors contradict themselves describing the observation as a phase transition. Moreover, according to Figure 3, there seem to be no phase transition in the case of $\mathrm{Na}+$, while there might be a bi-phasic behavior in the case of $\mathrm{K}+$.

Response: We are sorry about the confusion. Fig. 3a, 3b and 3c shows the diffraction peaks gradually shift to smaller angles during the intercalation of $\mathrm{Na}+$, indicating the continuous lattice expansion which is a characteristic of a solid solution mechanism. Only when the intercalation amount reaches 1.27, the fully discharged state, the framework cannot maintain and the (220) peak splits into two peaks, indicating a phase transition. This phenomenon was also observed in the system of organic phase and attribute to a phase change (J. Am. Chem. Soc., 137 (2015) 2548). We agree with the reviewer that there is no phase transition in the case of $\mathrm{Na}^{+}$while there might be a bi-phasic behavior for $\mathrm{K}^{+}$according to Figure 3, which is also in accordance with the understanding in our manuscript. We have modified the description in line 54-55 (left column) in page 3 as follows:

The XRD pattern of BG splits into two peaks (Figure S6) Only when $\mathrm{x}$ in $\mathrm{Na}_{\mathrm{x}} \mathrm{FeFe}(\mathrm{CN})_{6}$ reaches 1.27 , the (220) peak splits into two peaks becasue the framework of BG can not maintain. It indicates a phase transition from cubic to rhombohedral.

5. We don't agree with the conclusion about the unchanged $v(\mathrm{C} \equiv \mathrm{N})$ when using $\mathrm{K}_{2} \mathrm{SO}_{4}$. If you look at the region between 2100 and $2200 \mathrm{~cm}-1$, there is a band increasing in inteisty at ca. $2130 \mathrm{~cm}-1$ from the middle of the discharge. The strong peak at ca. $2170 \mathrm{~cm}-1$ shifts to lower frequencies when approaching the end of discharge. A much deeper and detailed interpretation on the modification of the $v(C \equiv N)$ vibration is expected.

Response: Thanks for pointing out this problem. The $\mathrm{E}_{\mathrm{g}}$ mode at ca. $2130 \mathrm{~cm}^{-1}$ appears and increases from the middle of the discharge process. The XRD patterns at the corresponded potential shown in Fig. $3 f$ also present an obvious change. Therefore, the emergence of Eg mode is resulted from the phase transition process during $\mathrm{K}^{+}$insertion. The peak at ca. $2170 \mathrm{~cm}^{-1}$ keeps unchanged until the end of discharge. As we described in line 9-11 (right volumn) in page 4, further splitting of the $\mathrm{T}_{1 \mathrm{u}}$ mode at the end of discharge indicates a lowering symmetry of the structure due to the strong interaction between $\mathrm{K}^{+}$and $\mathrm{C} \equiv \mathrm{N}$. This interaction leads to the shift of $v(\mathrm{C} \equiv \mathrm{N})$ vibration. We have modified the description in line 9-12 (right column) in page 4 as follows:

In the $\mathrm{K}_{2} \mathrm{SO}_{4}$ system, the $\mathrm{E}_{\mathrm{g}}$ mode at ca. $2130 \mathrm{~cm}^{-1}$ appears and increases from the middle of the discharge process, where the XRD patterns at the corresponded potential shown in Figure $3 \mathrm{f}$ also present an obvious change. Therefore, the $\mathrm{E}_{\mathrm{g}}$ mode may be resulted from local structure distortion during $\mathrm{K}^{+}$insertion. The triple degeneracy $\mathrm{T}_{1 \mathrm{u}}$ mode was further split into two peaks at $2096 \mathrm{~cm}^{-1}$ and $2053 \mathrm{~cm}^{-1}$ (Figure 5c), which indicates a lowering symmetry as a result of the strong interaction between $\mathrm{K}^{+}$and $\mathrm{C} \equiv \mathrm{N}$. The $\mathrm{A}_{1 \mathrm{~g}}$ mode at ca. $2170 \mathrm{~cm}^{-1}$ also shifts at the end of the discharge process due to this strong interaction.

6. The conclusion about that the $v^{2}(\mathrm{Fe}-\mathrm{C})$ is more sensitive than $v^{2}(\mathrm{C} \equiv \mathrm{N})$ conflicted with Figure 5a regarding to the case of $\mathrm{Na}^{+}$.

Response: Thanks for pointing out this problem, which is also mentioned in the comment 3 of reviewer 1. As stated in the response to reviewer 1 , because Fe is the redox center, it is more precise to say that $v$ 
${ }^{2}(\mathrm{Fe}-\mathrm{C})$ is more sensitive to the gain or loss of electron than the $v^{2}(\mathrm{C} \equiv \mathrm{N})$. We have modified the description in line 23-26 and line 48 to 53 (left column) in page 4 as follows:

We then plot the amount of intercalated cations with $v^{2}(\mathrm{Fe}-\mathrm{C})$ at $539 \mathrm{~cm}^{-1}$ to those of $v^{2}(\mathrm{C} \equiv \mathrm{N})$ at $2158 \mathrm{~cm}^{-1}$. As shown in Figure $5 \mathrm{a}$, the dependence of $v^{2}(\mathrm{Fe}-\mathrm{C})$ on the intercalation amount of cations in $\mathrm{Na}_{2} \mathrm{SO}_{4}$ is similar to that in $\mathrm{K}_{2} \mathrm{SO}_{4}$, while the dependence of $v^{2}(\mathrm{C} \equiv \mathrm{N})$ is obviously different. We propose that Fe in $\mathrm{BG}$ gains electrons first during the intercalation process, resulting in the shift of the $v(\mathrm{Fe}-\mathrm{C})$ peak. Since the gain or loss of electrons only relate to the change of bond order of $\mathrm{Fe}-\mathrm{C}$, the curves of $v(\mathrm{Fe}-\mathrm{C}) \mathrm{in}_{2} \mathrm{Na}_{2} \mathrm{SO}_{4}$ and $\mathrm{K}_{2} \mathrm{SO}_{4}$ overlap with each other well. After the ion intercalation, the lattice structure of $\mathrm{BG}$ changes. The different dependence of $v(\mathrm{C} \equiv \mathrm{N})$ suggests different intercalation mechanisms for $\mathrm{Na}^{+}$and $\mathrm{K}^{+}$.

7. We think that the authors should compare the Raman spectra of the pristine BG with those at the EOD (with $\mathrm{Na}^{+}$and $\mathrm{K}^{+}$) to better explain how the change in the symmetry influence on the mechanism.

Response: The Raman spectra for the electrochemical cycle from pristine BG to fully discharged state and the reverse process has been presented in Fig. 4 . The detailed analysis of the variation and the corresponded explanation are described in the last paragraph in page 3 and the first three paragraphs in page 4 .

8. The conclusion about volume expansion due to the $\mathrm{K}+$ insertion on page 6 , right column, line 51-52 conflicted with the operando XRD (Figure 3e,f), where we see the peaks shifting to the higher angles, which rather indicate a reduction of the lattice size.

Response: We are sorry that we did not describe it clearly. As shown in Fig. 3f, at the very beginning of insertion, the XRD peak shifts to lower angles, indicating an expansion of the framework. Afterwards, the lattice size decreases with the increased inserted amount of $\mathrm{K}^{+}$. Fortunately, as stated in the response to comment 2 of Reviewer 2, small amount of inserted $\mathrm{K}+$ serve as pillars to provide larger cell volume. Then $\mathrm{Na}^{+}$insertion will dominate to ensure a high specific capacity. We have modified the description in line 14 (right column) in page 3 as follows:

Different from that in $\mathrm{Na}_{2} \mathrm{SO}_{4}$, the potential-controlled electrochemical XRD study (Figure S7, Figure 3d, $3 \mathrm{~d}$ and $3 \mathrm{f}$ ) shows that the diffraction peaks move toward lower angles in $\mathrm{K}_{2} \mathrm{SO}_{4}$ at the very beginning of $\mathrm{K}+$ insertion and then move to higher angles with increasing inserted $\mathrm{K}^{+}$.

\section{Minor comments:}

1. According to the common scientific terminology, "intercalation" is the insertion of molecules or ions into a layered material. Since BG is not layered material, "insertion" should be used instead. This should be corrected everywhere in the paper.

Response: Thanks for the kind suggestion. Both "intercalation" and "insertion" are widely used in the literature. According to the definition of IUPAC (I03077), intercalation can refer to the insertion of a guest species into a one-, two- or three-dimensional host structure. A intercalation reaction, generally reversible, involves the penetration of a host material by guest species without causing a major structural modification of the host. Therefore, we here prefer to use "intercalation".

\section{In the Figures S5, S6, S8, separate the diffractograms as in Figure S7.}

Response: Thanks for the kind suggestion. The curves are stacked to show either the reversibility or variation after galvanostatic measurements. We used different color and line width to show different curves, enabling a clear comparison of both peak intensity and position. If separated, the comparison will not be so straightforward.

\section{References}


[1] M. Wang, H. Wang, H. Zhang, X. Li, Aqueous K-ion battery incorporating environment-friendly organic compound and Berlin green, J. Energy Chem. 48 (2020) 14-20. doi:10.1016/j.jechem.2019.12.019. [2] W. Shi, P. Nie, X. Shang, J. Yang, Z. Xie, R. Xu, J. Liu, Berlin green-based battery deionization-highly selective potassium recovery in seawater, Electrochim. Acta. 310 (2019) 104-112. doi:10.1016/j.electacta.2019.04.122.

[3] L. Samain, B. Gilbert, F. Grandjean, G.J. Long, D. Strivay, Redox reactions in Prussian blue containing paint layers as a result of light exposure, J. Anal. At. Spectrom. 28 (2013) 524. doi:10.1039/c3ja30359d. 